(C)2007 IEEE. Personal use of this material is permitted. However, permission to reprint/republish this material for advertising or promotional purposes or for creating new collective works for resale or redistribution to servers or lists, or to reuse any copyrighted component of this work in other works must be obtained from the IEEE. 


\title{
On the Partial Realization of Noncausal 2-D Linear Systems
}

\author{
Lorenzo Ntogramatzidis, Michael Cantoni, Member, IEEE, and Ran Yang
}

\begin{abstract}
The problem of partial realization is to construct a latent variable model which matches a specified input-output behavior over a bounded frame of interest. In this paper, an algorithm is proposed for constructing a partial realization from the Toeplitz kernel of a possibly noncausal 2-D linear system. By construction, the resulting latent variable model and corresponding boundary conditions comprise four components, each with recursively computable structure.
\end{abstract}

Index Terms-Noncausal 2-D systems, nearest neighbour models (NNMs), partial realization.

\section{INTRODUCTION}

B ROADLY, a two-dimensional (2-D) system relates signals indexed by two free variables. In many situations, these free variables represent discrete spatial co-ordinates; e.g., image processing and the study of spatially distributed processes [3], [11]. Various classes of latent variable models have found application in the study of linear discrete 2-D systems, including: the inherently quarter-plane causal models of Roesser [23] and Fornasini-Marchesini (FM) [6], [7]; the implicit generalizations of Roesser and FM models [12], [19], [17], which are capable of generating noncausal input-output behavior; and the so-called nearest neighbour model (NNM) of [16], which is also capable of accommodating noncausal relationships between inputs and outputs.

The task of constructing a latent variable model to match the infinite horizon behavior of a specified input-output model is known as the realization problem. This problem has received considerable attention [6], [14], [4], [10], [2], [24] for so-called quarter-plane causal 2-D systems, with the resulting realization being invariably in the form of a (recursive) Roesser or FM model. The realization problem for 2-D system without quarterplane causal input-output behavior in terms of implicit generalizations of these models, however, has received relatively little attention [5], [25]. Similarly, to the best of the authors' knowledge, the realization of an NNM latent variable model from a given input-output representation has not been considered. Indeed, much of the literature on the realization of noncausal 2-D

Manuscript received December 7, 2006. This work was supported in part by the the Australian Research Council under Grant DP0664789. This paper was recommended by Associate Editor A. Kummert.

L. Ntogramatzidis and M. Cantoni are with the Department of Electrical and Electronic Engineering, The University of Melbourne, Parkville, VIC 3010, Australia (e-mail: Int@ee.unimelb.edu.au; m.cantoni@ee.unimelb.edu.au).

R. Yang is with the School of Information Science and Technology, Sun Yat-Sen University, Guangzhou 510275, China (e-mail: yangran@mail.sysu. edu.cn).

Digital Object Identifier 10.1109/TCSI.2007.902543 processes [8], [26] is set in the behavioral framework of Willems [22], where a latent variable model is sought to describe the so-called behavior, comprising the inputs and outputs together as the entity of interest. This is quite different to the approach taken here where the partition of the behavior into inputs and outputs is pre-defined, as may be appropriate in various applications.

In this paper, the problem of partial realization is considered for noncausal 2-D processes, whereby it is only required to match the specified input-output behavior over a bounded frame of interest. In particular, an algorithm is proposed for the construction of a latent variable model in NNM form to match (over a bounded frame) the input-output behavior of a 2-D linear system with specified Toeplitz kernel. First, it is observed that such a system can be naturally decomposed into four quarter-plane causal components. Indeed, via simple transformations each component is shown to be equivalent to a so-called south-west (sw) causal system. The focus then turns to the construction of what can be thought of as a rational polynomial model given a Toeplitz kernel with sw causal structure, by which a recursive latent variable model can be subsequently obtained. Finally, it is shown how to transform and combine the latent variable model realizations for each quarter-plane causal component of the original (noncausal) Toeplitz kernel into a single NNM model with appropriately assigned latent variable boundary conditions.

\section{INPUT-OUTPUT MODELS}

Let $N, M \in \mathbb{N} \backslash\{0\}$. Given two intervals $[0, N]$ and $[0, M]$ of $\mathbb{N}$, consider the bounded-frame linear process $\mathcal{F}: u \mapsto y$, governed by

$$
y_{k, l}=\sum_{i=0}^{N} \sum_{j=0}^{M} \varphi_{k-i, l-j} u_{i, j}, \quad(k, l) \in[0, N] \times[0, M]
$$

where $u, y:[0, N] \times[0, M] \rightarrow \mathbb{R}$ represent the input and the output of the system, respectively, and $\varphi:[-N, N] \times$ $[-M, M] \rightarrow \mathbb{R}$ is the so-called Toeplitz kernel (or impulse response) of $\mathcal{F}$. Note that (1) does not impose a causal relationship between the input $u$ and the output $y$. However, the following forms of quarter-plane causality play an important role in the approach to realization described in this paper.

- South-West (sw) —at any point $(k, l) \in[0, N] \times[0, M]$, the output $y_{k, l}$ depends only on values of the input $u_{i, j}$ for $i \leq k$ and $j \leq l$.

- North-West (nw) - at any point $(k, l) \in[0, N] \times[0, M]$, the output $y_{k, l}$ depends only on values of the input $u_{i, j}$ for $i \leq k$ and $j \geq l$. 
TABLE I

DEFINITIONS OF INTERVALS DEPENDING ON THE SYMBOL $\Delta$

\begin{tabular}{|c||c|c||c|c|}
\hline$\Delta$ & $I_{h}^{\Delta}$ & $I_{v}^{\Delta}$ & $H_{k}^{\Delta}$ & $V_{l}^{\Delta}$ \\
\hline \hline sw & $(0, N]$ & $(0, M]$ & {$[0, k]$} & {$[0, l]$} \\
\hline nw & $(0, N]$ & {$[-M, 0)$} & {$[0, k]$} & {$[l, M]$} \\
\hline ne & {$[-N, 0)$} & {$[-M, 0)$} & {$[k, N]$} & {$[l, M]$} \\
\hline se & {$[-N, 0)$} & $(0, M]$ & {$[k, N]$} & {$[0, l]$} \\
\hline
\end{tabular}

- North-East (ne)—at any point $(k, l) \in[0, N] \times[0, M]$, the output $y_{k, l}$ depends only on values of the input $u_{i, j}$ for $i \geq k$ and $j \geq l$.

- South-East (se) —at any point $(k, l) \in[0, N] \times[0, M]$, the output $y_{k, l}$ depends only on values of the input $u_{i, j}$ for $i \geq k$ and $j \leq l$.

The causal structure of $\mathcal{F}$ is said to be strict in the horizontal (respectively, vertical) direction if the inequality on the index $i$ (respectively, $j$ ) is strict. For example, if at any point $(k, l) \in$ $[0, N] \times[0, M]$ the output $y_{k, l}$ depends only on values of the input $u_{i, j}$ for $i \leq k$ and $j>l$, the nw causal dependence of $y$ on $u$ is said to be strict in the vertical direction.

In what follows, it is shown that an arbitrary bounded-frame system of the form (1) can be linearly decomposed into four components, each exhibiting one of the aforementioned causal dependencies on the input. In particular, define the symbol $\Delta \epsilon$ \{sw, nw, ne, se\}, and let $I_{h}^{\Delta}$ and $I_{v}^{\Delta}$ be defined as shown in Table I. Furthermore, for all $\Delta \in\{\mathrm{sw}, \mathrm{nw}, \mathrm{ne}, \mathrm{se}\}$ let

$$
\varphi_{k, l}^{\Delta}:= \begin{cases}\varphi_{k, l}, & (k, l) \in I_{h}^{\Delta} \times I_{v}^{\Delta} \\ \frac{1}{2} \varphi_{k, l}, & (k, l) \in\left(\{0\} \times I_{v}^{\Delta}\right) \\ & \cup\left(I_{h}^{\Delta} \times\{0\}\right) \backslash\{(0,0)\} \\ \frac{1}{4} \varphi_{k, l}, & (k, l)=(0,0) \\ 0, & \text { otherwise. }\end{cases}
$$

Then, (1) can be rewritten as the sum of four components

$$
\begin{aligned}
y_{k, l} & =\sum_{i=0}^{k} \sum_{j=0}^{l} \varphi_{k-i, l-j}^{\mathrm{sw}} u_{i, j} \\
& +\sum_{i=0}^{k} \sum_{j=l}^{M} \varphi_{k-i, l-j}^{\mathrm{nw}} u_{i, j} \\
& +\sum_{i=k}^{N} \sum_{j=l}^{M} \varphi_{k-i, l-j}^{\mathrm{ne}} u_{i, j} \\
& +\sum_{i=k}^{N} \sum_{j=0}^{l} \varphi_{k-i, l-j}^{\mathrm{se}} u_{i, j} .
\end{aligned}
$$

Moreover, with $H_{k}^{\Delta}$ and $V_{l}^{\Delta}$ defined as in Table I, it follows that $y_{k, l}=\sum_{\Delta \in\{\mathrm{sw}, \mathrm{nw}, \mathrm{ne}, \mathrm{se}\}} y_{k, l}^{\Delta}$, where

$$
y_{k, l}^{\Delta}:=\sum_{i \in H_{k}^{\Delta}} \sum_{j \in V_{l}^{\Delta}} \varphi_{k-i, l-j}^{\Delta} u_{i, j}
$$

for all $\Delta \in\{\mathrm{sw}, \mathrm{nw}, \mathrm{ne}, \mathrm{se}\}$. Note that the dependence of each $y^{\Delta}$ on the input $u$ is consistent with a corresponding $\Delta$ quarterplane causality. Indeed, the system $\mathcal{F}^{\Delta}: u \mapsto y^{\Delta}$ described by (4) is $\Delta$-causal.

Interestingly, a simple transformation of the spatial indexes permits re-statement of (4) in terms of a convolution with
TABLE II

DEFINITIONS OF INDEXES AND SIGN CONSTANTS DEPENDING ON $\Delta$

\begin{tabular}{|c||c|c||c|c||}
\hline$\Delta$ & $i_{\Delta}$ & $j_{\Delta}$ & $s_{h}^{\Delta}$ & $s_{v}^{\Delta}$ \\
\hline \hline sw & $i$ & $j$ & 1 & 1 \\
\hline nw & $i$ & $M-j$ & 1 & -1 \\
\hline ne & $N-i$ & $M-j$ & -1 & -1 \\
\hline se & $N-i$ & $j$ & -1 & 1 \\
\hline
\end{tabular}

sw-causal structure. In particular, for each $\Delta \in\{\mathrm{sw}, \mathrm{nw}, \mathrm{ne}, \mathrm{se}\}$ and $(i, j) \in[0, N] \times[0, M]$, define $\hat{u}_{i, j}^{\Delta}:=u_{i_{\Delta}, j_{\Delta}}$ and $\hat{\varphi}_{i, j}^{\Delta}:=\varphi_{s_{h}^{\Delta} i, s_{v}^{\Delta} j}^{\Delta}$, where $i_{\Delta}, j_{\Delta}, s_{v}^{\Delta}$ and $s_{h}^{\Delta}$ are given in Table II. Then each $\hat{\varphi}^{\Delta}$ is nonzero only in the bounded frame of interest $[0, N] \times[0, M]$ and the system $\hat{u}^{\Delta} \mapsto \hat{y}^{\Delta}$ defined by

$$
\hat{y}_{k, l}^{\Delta}:=\sum_{(i, j) \in[0, k] \times[0, l]} \hat{\varphi}_{k-i, l-j}^{\Delta} \hat{u}_{i, j}^{\Delta}
$$

for $(k, l) \in[0, N] \times[0, M]$, has sw-causal structure and satisfies $y_{i, j}^{\Delta}=\hat{y}_{i_{\Delta}, j_{\Delta}}^{\Delta}$, for all $(i, j) \in[0, N] \times[0, M]$. Correspondingly, an approach to constructing a realization for (1) is to first realize each sw-causal counterpart (5) of (3), followed by inversion of the associated spatial index transformations and subsequent combination of the resulting latent variable models and boundary conditions, as discussed in Sections III and IV, respectively, see also [21].

Remark 2.1: Note that (2) is not the only decomposition of the Toeplitz kernel $\varphi$ for which this approach is possible. For example, with

$$
\begin{aligned}
& \varphi_{k, l}^{\mathrm{sw}}:=\varphi_{k, l} \quad(k, l) \in[0, N] \times[0, M] \\
& \varphi_{k, l}^{\mathrm{nw}}:= \begin{cases}\varphi_{k, l} & (k, l) \in[0, N] \times[-M, 0) \\
0 & (k, l) \in[0, N] \times\{0\}\end{cases} \\
& \varphi_{k, l}^{\text {ne }}:= \begin{cases}\varphi_{k, l} & (k, l) \in[-N, 0) \times[-M, 0) \\
0 & (k, l) \in([-N, 0] \times\{0\}) \cup(\{0\} \times[-M, 0])\end{cases} \\
& \varphi_{k, l}^{\mathrm{se}}:= \begin{cases}\varphi_{k, l} & (k, l) \in[-N, 0) \times[0, M] \\
0 & (k, l) \in\{0\} \times[0, M]\end{cases}
\end{aligned}
$$

the decomposition in (3) still holds. In this case, the dependencies of the outputs $y^{\mathrm{nw}}, y^{\mathrm{ne}}$ and $y^{\text {se }}$ thus obtained, on the input $u$, are strict in the vertical direction, in both the vertical and horizontal direction, and in the horizontal direction, respectively.

\section{Rational Polynomial Models and Partial REALIZATION}

In view of the considerations above, the aim in this section is to define and solve a partial realization problem, given a sw-causal process $\mathcal{F}: u \mapsto y$ described by

$$
y_{k, l}=\sum_{i=0}^{k} \sum_{j=0}^{l} \varphi_{k-i, l-j} u_{i, j}
$$

for $(k, l) \in[0, N] \times[0, M]$; since $\mathcal{F}$ is sw-causal, $\varphi_{i, j}=0$ for all $i, j$ such that $i<0$ or $j<0$. To this end, the partial realization problem is first formulated in terms of the existence of a rational polynomial model, where the representation of a 
doubly indexed signal in terms of a formal power series is exploited; given a signal $s: \mathbb{N} \times \mathbb{N} \rightarrow \mathbb{R}$,

$$
\mathbf{s}\left(\lambda_{h}, \lambda_{v}\right):=\sum_{(i, j) \in \mathbb{N} \times \mathbb{N}} s_{i, j} \lambda_{h}^{i} \lambda_{v}^{j} \in \mathbb{R}\left[\left[\lambda_{h}, \lambda_{v}\right]\right]
$$

where the indeterminates $\lambda_{h}$ and $\lambda_{v}$ may be viewed as markers of the spatial index $(i, j)$ and $\mathbb{R}\left[\left[\lambda_{h}, \lambda_{v}\right]\right]$ denotes the ring of formal power series in the two indeterminates $\lambda_{h}$ and $\lambda_{v}$.

As is well known and easily established [20], the convolution of $u$ and $\varphi$ in (6) can be represented by the product of the respective formal power series. Indeed, with the truncation operator $\Pi_{I_{h} \times I_{v}}: \mathbb{R}\left[\left[\lambda_{h}, \lambda_{v}\right]\right] \rightarrow \mathbb{R}\left[\left[\lambda_{h}, \lambda_{v}\right]\right]$ defined so that $\Pi_{I_{h} \times I_{v}}\left(\mathbf{s}\left(\lambda_{h}, \lambda_{v}\right)\right)=\sum_{(i, j) \in I_{h} \times I_{v}} s_{i, j} \lambda_{h}^{i} \lambda_{v}^{j}$ for intervals $I_{h}$ and $I_{v}$ of $\mathbb{N}$, the input-output relation (6) can be written as

$$
\mathbf{y}\left(\lambda_{h}, \lambda_{v}\right)=\Pi_{[0, N] \times[0, M]}\left(\varphi\left(\lambda_{h}, \lambda_{v}\right) \mathbf{u}\left(\lambda_{h}, \lambda_{v}\right)\right)
$$

where $\mathbf{u}\left(\lambda_{h}, \lambda_{v}\right), \varphi\left(\lambda_{h}, \lambda_{v}\right)$ and $\mathbf{y}\left(\lambda_{h}, \lambda_{v}\right)$ are the bivariate polynomials corresponding to the formal power-series of the natural extensions (zero padding) of $u, \varphi$ and $y$ to $\mathbb{N} \times \mathbb{N}$, respectively. This naturally leads to the following partial realization problem, from which a corresponding latent variable model can be constructed as described in Section III-B.

Problem 3.1: Given $\varphi\left(\lambda_{h}, \lambda_{v}\right)$, find $(n, m) \in[0, N] \times[0, M]$ and polynomials

$$
\begin{aligned}
\mathbf{p}\left(\lambda_{h}, \lambda_{v}\right) & :=\sum_{i=0}^{n} \sum_{j=0}^{m} p_{i, j} \lambda_{h}^{i} \lambda_{v}^{j} \quad \text { with } p_{0,0}=1 \\
\mathbf{q}\left(\lambda_{h}, \lambda_{v}\right) & :=\sum_{i=0}^{n} \sum_{j=0}^{m} q_{i, j} \lambda_{h}^{i} \lambda_{v}^{j}
\end{aligned}
$$

such that the identity

$$
\mathbf{p}\left(\lambda_{h}, \lambda_{v}\right)\left(\varphi\left(\lambda_{h}, \lambda_{v}\right)+\gamma\left(\lambda_{h}, \lambda_{v}\right)\right)=\mathbf{q}\left(\lambda_{h}, \lambda_{v}\right)
$$

holds for some

$$
\boldsymbol{\gamma}\left(\lambda_{h}, \lambda_{v}\right)=\sum_{(i, j) \in \mathbb{N} \times \mathbb{N} \backslash[0, N] \times[0, M]} \gamma_{i, j} \lambda_{h}^{i} \lambda_{v}^{j} \in \mathbb{R}\left[\left[\lambda_{h}, \lambda_{v}\right]\right]
$$

For polynomials $\mathbf{p}\left(\lambda_{h}, \lambda_{v}\right)$ and $\mathbf{q}\left(\lambda_{h}, \lambda_{v}\right)$ such that (9) holds with an appropriate $\boldsymbol{\gamma}\left(\lambda_{h}, \lambda_{v}\right)$, it follows immediately that given any $u$ and $y$ that satisfy (6)

$$
\mathbf{p}\left(\lambda_{h}, \lambda_{v}\right) \mathbf{z}\left(\lambda_{h}, \lambda_{v}\right)=\mathbf{q}\left(\lambda_{h}, \lambda_{v}\right) \mathbf{u}\left(\lambda_{h}, \lambda_{v}\right)
$$

for a $\mathbf{z}\left(\lambda_{h}, \lambda_{v}\right)$ that satisfies $\Pi_{[0, N] \times[0, M]}\left(\mathbf{z}\left(\lambda_{h}, \lambda_{v}\right)\right)=$ $\mathbf{y}\left(\lambda_{h}, \lambda_{v}\right)$. That is, $\mathbf{p}\left(\lambda_{h}, \lambda_{v}\right)$ and $\mathbf{q}\left(\lambda_{h}, \lambda_{v}\right)$ constitute a rational model that matches the input-output behavior of (6) over the bounded frame $[0, N] \times[0, M]$. It is without loss of generality that the degree $(n, m)$ of the polynomials $\mathbf{p}\left(\lambda_{h}, \lambda_{v}\right)$ and $\mathbf{q}\left(\lambda_{h}, \lambda_{v}\right)$ is restricted to $[0, N] \times[0, M]$, since (9) holds with $\boldsymbol{\gamma}\left(\lambda_{h}, \lambda_{v}\right)=0$ by taking $\mathbf{p}\left(\lambda_{h}, \lambda_{v}\right)=p_{0,0}=1$ and $\mathbf{q}\left(\lambda_{h}, \lambda_{v}\right)=\varphi\left(\lambda_{h}, \lambda_{v}\right)$, in which case the degree of $\mathbf{q}\left(\lambda_{h}, \lambda_{v}\right)$ is $(N, M)$. This (trivial) solution, however, does not exploit the degree of freedom in $\boldsymbol{\gamma}\left(\lambda_{h}, \lambda_{v}\right)$, which can be used to keep the degrees of both $\mathbf{p}\left(\lambda_{h}, \lambda_{v}\right)$ and $\mathbf{q}\left(\lambda_{h}, \lambda_{v}\right)$ small in some sense. This is important for the construction of a corresponding latent variable model, as described in Section III-B.

\section{A. Characterising Solutions of Problem 3.1}

Towards characterising solutions of Problem 3.1, an equivalent formulation is first established in the following lemma.

Lemma 3.1: Problem 3.1 is equivalent to finding polynomials $\mathbf{p}\left(\lambda_{h}, \lambda_{v}\right)$ and $\mathbf{q}\left(\lambda_{h}, \lambda_{v}\right)$ in the form (7)-(8) such that the identity

$$
\mathbf{p}\left(\lambda_{h}, \lambda_{v}\right) \varphi\left(\lambda_{h}, \lambda_{v}\right)=\mathbf{q}\left(\lambda_{h}, \lambda_{v}\right)+\bar{\gamma}\left(\lambda_{h}, \lambda_{v}\right)
$$

holds for some

$$
\overline{\boldsymbol{\gamma}}\left(\lambda_{h}, \lambda_{v}\right):=\sum_{(i, j) \in \mathbb{N} \times \mathbb{N} \backslash[0, N] \times[0, M]} \bar{\gamma}_{i, j} \lambda_{h}^{i} \lambda_{v}^{j}
$$

with each $\bar{\gamma}_{i, j} \in \mathbb{R}$.

Proof: The existence of a $\boldsymbol{\gamma}\left(\lambda_{h}, \lambda_{v}\right)$ such that (9) holds implies the existence of a $\overline{\boldsymbol{\gamma}}\left(\lambda_{h}, \lambda_{v}\right)$ such that (10) holds because each element of the product $\mathbf{p}\left(\lambda_{h}, \lambda_{v}\right) \gamma\left(\lambda_{h}, \lambda_{v}\right)$ is a monomial of the form $\lambda_{h}^{\nu} \lambda_{v}^{\mu}$, with $\nu>N$ or $\mu>M$. Similarly, if an appropriate $\bar{\gamma}\left(\lambda_{h}, \lambda_{v}\right)$ exists such that (10) is satisfied, then (9) holds with

$$
\begin{aligned}
\boldsymbol{\gamma}\left(\lambda_{h}, \lambda_{v}\right) & =-\sum_{i \in \mathbb{N}}\left(-\overline{\mathbf{p}}\left(\lambda_{h}, \lambda_{v}\right)\right)^{i} \bar{\gamma}\left(\lambda_{h}, \lambda_{v}\right) \\
& =\sum_{(i, j) \in \mathbb{N} \times \mathbb{N} \backslash[0, N] \times[0, M]} \gamma_{i, j} \lambda_{h}^{i} \lambda_{v}^{j}
\end{aligned}
$$

since $\overline{\mathbf{p}}\left(\lambda_{h}, \lambda_{v}\right):=\mathbf{p}\left(\lambda_{h}, \lambda_{v}\right)-1$ has no constant terms and

$$
\mathbf{p}\left(\lambda_{h}, \lambda_{v}\right) \sum_{i \in \mathbb{N}}\left(-\overline{\mathbf{p}}\left(\lambda_{h}, \lambda_{v}\right)\right)^{i}=1 .
$$

This completes the proof.

In what follows, it is shown how (10) can be expressed in a form that helps in defining computationally tractable solvability conditions for Problem 3.1, given values for $n \leq N$ and $m \leq$ $M$. More specifically, given a kernel $\varphi:[0, N] \times[0, M] \rightarrow \mathbb{R}$, and for $(i, j) \in[0, N] \times[0, M]$, let the $(N+1) \times(M+1)$ matrix $\Phi_{i, j}$ be defined by

$$
\Phi_{i, j}:=\left[\begin{array}{cc}
\Psi_{i, j} & 0_{(N-i+1) \times j} \\
0_{i \times(M-j+1)} & 0_{i \times j}
\end{array}\right]
$$

where

$$
\Psi_{i, j}:=\left[\begin{array}{ccc}
\varphi_{N-i, M-j} & \ldots & \varphi_{N-i, 0} \\
\vdots & \ddots & \vdots \\
\varphi_{0, M-j} & \cdots & \varphi_{0,0}
\end{array}\right]
$$


is a $(N-i+1) \times(M-j+1)$ matrix. Now given a polynomial $\mathbf{p}\left(\lambda_{h}, \lambda_{v}\right)$,

$$
\begin{aligned}
& \mathbf{p}\left(\lambda_{h}, \lambda_{v}\right) \varphi\left(\lambda_{h}, \lambda_{v}\right) \\
& =\sum_{i=0}^{n} \sum_{j=0}^{m} p_{i, j} \lambda_{h}^{i} \lambda_{v}^{j} \sum_{k=0}^{N} \sum_{l=0}^{M} \varphi_{k, l} \lambda_{h}^{k} \lambda_{v}^{l} \\
& =\sum_{i=0}^{n} \sum_{j=0}^{m} p_{i, j} \sum_{\bar{k}=i}^{N+i} \sum_{\bar{l}=j}^{M+j} \varphi_{\bar{k}-i, \bar{l}-j} \lambda_{h}^{\bar{k}} \lambda_{v}^{\bar{l}} \\
& =\sum_{i=0}^{n} \sum_{j=0}^{m} p_{i, j}\left(\sum_{\bar{k}=i}^{N} \sum_{\bar{l}=j}^{M} \varphi_{\bar{k}-i, \bar{l}-j} \lambda_{h}^{\bar{k}} \lambda_{v}^{\bar{l}}\right. \\
& \left.\quad+\boldsymbol{\eta}_{i, j}\left(\lambda_{h}, \lambda_{v}\right)\right)
\end{aligned}
$$

where $\boldsymbol{\eta}_{i, j}\left(\lambda_{h}, \lambda_{v}\right):=\sum_{k=N+1}^{N+i} \sum_{l=j}^{M+j} \varphi_{k-i, l-j} \lambda_{h}^{k} \lambda_{v}^{l}+$ $\sum_{k=i}^{N} \sum_{l=M+1}^{M+j} \varphi_{k-i, l-j} \lambda_{h}^{k} \lambda_{v}^{l}$. Moreover, since

$$
\begin{aligned}
\sum_{\bar{k}=i}^{N} & \sum_{\bar{l}=j}^{M} \varphi_{\bar{k}-i, \bar{l}-j} \lambda_{h}^{\bar{k}} \lambda_{v}^{\bar{l}} \\
= & {\left[\begin{array}{lllll}
\lambda_{h}{ }^{N} & \lambda_{h}{ }^{N-1} & \ldots & \lambda_{h}{ }^{i}
\end{array}\right] \Psi_{i, j} } \\
& \times\left[\begin{array}{llll}
\lambda_{v}{ }^{M} & \lambda_{v}{ }^{M-1} & \ldots & \lambda_{v}{ }^{j}
\end{array}\right]^{\top} \\
= & \Lambda_{h} \Phi_{i, j} \Lambda_{v}^{\top}
\end{aligned}
$$

where $\Lambda_{h} \quad:=\left[\begin{array}{llll}\lambda_{h}^{N} & \ldots & \lambda_{h} & 1\end{array}\right]$ and $\Lambda_{v} \quad:=$ $\left[\begin{array}{llll}\lambda_{v}^{M} & \ldots & \lambda_{v} & 1\end{array}\right], \quad$ it follows that the product $\mathbf{p}\left(\lambda_{h}, \lambda_{v}\right) \varphi\left(\lambda_{h}, \lambda_{v}\right)$ can be written as the sum

$$
\mathbf{p}\left(\lambda_{h}, \lambda_{v}\right) \varphi\left(\lambda_{h}, \lambda_{v}\right)=\Lambda_{h} \mathbf{Q} \Lambda_{v}^{\top}+\bar{\gamma}\left(\lambda_{h}, \lambda_{v}\right)
$$

where

$$
\mathbf{Q}:=\sum_{i=0}^{n} \sum_{j=0}^{m} p_{i, j} \Phi_{i, j}
$$

and

$$
\begin{aligned}
\bar{\gamma}\left(\lambda_{h}, \lambda_{v}\right) & :=\sum_{i=0}^{n} \sum_{j=0}^{m} p_{i, j} \boldsymbol{\eta}_{i, j}\left(\lambda_{h}, \lambda_{v}\right) \\
& =\sum_{(i, j) \in \mathbb{N} \times \mathbb{N} \backslash[0, N] \times[0, M]} \bar{\gamma}_{i, j} \lambda_{h}^{i} \lambda_{v}^{j} .
\end{aligned}
$$

Equation (11) is now utilized to establish conditions for the existence of a solution $\mathbf{p}\left(\lambda_{h}, \lambda_{v}\right)$ and $\mathbf{q}\left(\lambda_{h}, \lambda_{v}\right)$ for Problem 3.1, given values for $n \leq N$ and $m \leq M$. By comparing (11) with (10) it is clear that if (11) holds, the polynomials $\mathbf{p}\left(\lambda_{h}, \lambda_{v}\right)$ and $\mathbf{q}\left(\lambda_{h}, \lambda_{v}\right)=\Lambda_{h} \mathbf{Q} \Lambda_{v}^{\top}$ are a solution for Problem 3.1 if, and only if, the degree of $\Lambda_{h} \mathrm{Q} \Lambda_{v}^{\top}$ is (componentwise) no greater than $(n, m)$. The condition which the coefficients of $\mathbf{p}\left(\lambda_{h}, \lambda_{v}\right)$ must satisfy for this to happen is that the matrix $\mathbf{Q}=\sum_{i=0}^{n} \sum_{j=0}^{m} p_{i, j} \Phi_{i, j}$ have the first $N-n$ rows and the first $M-m$ columns equal to zero: i.e.,

$$
\mathbf{Q}=\left[\begin{array}{cc}
0_{(N-n) \times(M-m)} & 0_{(N-n) \times(m+1)} \\
0_{(n+1) \times(M-m)} & \boldsymbol{\Omega}
\end{array}\right]
$$

for some $\Omega \in \mathbb{R}^{(n+1) \times(m+1)}$. In fact, in this case

$$
\begin{aligned}
& \mathbf{q}\left(\lambda_{h}, \lambda_{v}\right)=\Lambda_{h} \mathbf{Q} \Lambda_{v}^{\top}=\left[\begin{array}{llll}
\lambda_{h}^{n} & \ldots & \lambda_{h} & 1
\end{array}\right] \Omega \\
& \times\left[\begin{array}{lllll}
\lambda_{v}^{m} & \ldots & \lambda_{v} & 1
\end{array}\right]^{\top}
\end{aligned}
$$

which is of the required form (8). In summary, we have the following lemma.

Lemma 3.2: Given $n \leq N$ and $m \leq M$, there exist polynomials $\mathbf{p}\left(\lambda_{h}, \lambda_{v}\right)$ and $\mathbf{q}\left(\lambda_{h}, \lambda_{v}\right)$ with the form specified in (7)-(8), that partially realize the kernel $\varphi$ (i.e., solve Problem $3.1)$ if and only if $[\mathrm{Q}]_{i, j}=0$ for $i=1, \ldots, N-n$ and $j=1, \ldots, M-m$, where $[\mathbf{Q}]_{i, j}$ denotes the $(i, j)$ th entry of the matrix $\mathbf{Q}$ defined in (12).

Now, denoting by $R_{k}^{(i, j)}$ and $C_{l}^{(i, j)}$ the $(k+1)$ th row and the $(l+1)$ th column of $\Phi_{i, j}$, respectively, $\mathbf{Q}$ can be written as

$$
\begin{aligned}
\mathbf{Q}= & \sum_{i=0}^{n} \sum_{j=0}^{m} p_{i, j} \\
& \times\left[\begin{array}{llll}
\left(R_{0}^{(i, j)}\right)^{\top} & \left(R_{1}^{(i, j)}\right)^{\top} & \ldots & \left(R_{N}^{(i, j)}\right)^{\top}
\end{array}\right]^{\top} \\
= & \sum_{i=0}^{n} \sum_{j=0}^{m} p_{i, j}\left[\begin{array}{llll}
C_{0}^{(i, j)} & C_{1}^{(i, j)} & \ldots & C_{M}^{(i, j)}
\end{array}\right] .
\end{aligned}
$$

Then with $k_{0}:=N-n-1$ and $l_{0}:=M-m-1,{ }^{1}$ it follows that if $n<N$ (i.e., if $k_{0} \geq 0$ ), the first $k_{0}+1$ rows of $\mathbf{Q}$ are zero if, and only if

$$
\sum_{i=0}^{n} \sum_{j=0}^{m} p_{i, j} R_{k}^{(i, j)}=0
$$

holds for all $k=0,1, \ldots, k_{0}$. When $n=N$ (i.e., $k_{0}=-1$ ), (16) need not be imposed. Similarly, when $l_{0} \geq 0$, the first $l_{0}+1$ columns of $\mathbf{Q}$ are zero if, and only if, for all $l=0,1, \ldots, l_{0}$ the identity

$$
\sum_{i=0}^{n} \sum_{j=0}^{m} p_{i, j} C_{l}^{(i, j)}=0
$$

holds, while when $m=M$ there is no need for (17). That is, (16) and (17) constitute a set of conditions that the coefficients of $\mathbf{p}\left(\lambda_{h}, \lambda_{v}\right)$ must satisfy in order for the first $k_{0}+1=N-n$ rows and $l_{0}+1=M-m$ columns of $\mathbf{Q}$ to be zero, as in (14). In particular, with $h^{(0,0)}\left(k_{0}, l_{0}\right)$ and $H_{[n, m]}\left(k_{0}, l_{0}\right)$ defined as shown at the bottom of the next page, the following computable necessary and sufficient condition for the solvability of Problem 3.1 is obtained. Note that the theorem statement includes an explicit way of computing the coefficients of the polynomial $\mathbf{p}\left(\lambda_{h}, \lambda_{v}\right)$, when the condition is feasible. Furthermore, observe that the matrix $H_{[n, m]}(k, l)$ is related to the 2-D Hankel matrix of [13].

Theorem 3.1: Given $n \leq N$ and $m \leq M$, there exists a polynomial $\mathbf{p}\left(\lambda_{h}, \lambda_{v}\right)$ of the form (7) such that the corresponding matrix $\mathbf{Q}$ in (12) has zeros in the first $k_{0}+1=N-n$ rows and the first $l_{0}+1=M-m$ columns if, and only if

$$
h^{(0,0)}\left(k_{0}, l_{0}\right) \in \operatorname{im} H_{[n, m]}\left(k_{0}, l_{0}\right) .
$$

${ }^{1}$ Note that $k_{0} \geq-1$ and $l_{0} \geq-1$, since $n \leq N$ and $m \leq M$. 
In this case, the vector of (free) coefficients ${ }^{2} \pi:=$ $\left[\begin{array}{lllll}p_{0,1} & p_{1,0} & \cdots & \cdots & p_{n, m}\end{array}\right]^{\top}$ for any polynomial $\mathbf{p}\left(\lambda_{h}, \lambda_{v}\right)$ that solves Problem 3.1 with the corresponding polynomial $\mathbf{q}\left(\lambda_{h}, \lambda_{v}\right)=\Lambda_{h} \mathbf{Q} \Lambda_{v}^{\top}$, can be expressed as

$$
\pi=-H_{[n, m]}\left(k_{0}, l_{0}\right)^{\dagger} h^{(0,0)}\left(k_{0}, l_{0}\right)+v
$$

for some $v \in \operatorname{ker} H_{[n, m]}\left(k_{0}, l_{0}\right)$, where the symbol $\dagger$ denotes the Moore-Penrose pseudoinverse.

Proof: Since $p_{0,0}=1$ in (7), conditions (16)-(17) are satisfied for the vector $\pi$ of the remaining coefficients for $\mathbf{p}\left(\lambda_{h}, \lambda_{v}\right)$ and all $0 \leq l \leq l_{0}$ and $0 \leq k \leq k_{0}$ if, and only if, $h^{(0,0)}\left(k_{0}, l_{0}\right)=-H_{[n, m]}\left(k_{0}, l_{0}\right) \pi$. Correspondingly, $\mathbf{Q}$ has the structure shown in (14) (with $\Omega$ having $N-k_{0}$ rows and $M-l_{0}$ columns) if, and only if, condition (18) holds. In this case, $\pi$ can be expressed as in (19) for some $v \in \operatorname{ker} H_{[n, m]}\left(k_{0}, l_{0}\right)$. Furthermore, by Lemma 3.2, the corresponding polynomial $\mathbf{p}\left(\lambda_{h}, \lambda_{v}\right)$ solves Problem 3.1 with $\mathbf{q}\left(\lambda_{h}, \lambda_{v}\right)=\Lambda_{h} \mathbf{Q} \Lambda_{v}^{\top}$.

Theorem 3.1 provides the basis for an algorithm to construct a partial realization of a Toeplitz kernel with sw causal structure. In particular, given a total ordering $\mathfrak{R}$ over $[0, N] \times[0, M] \backslash$ $\{(0,0)\}$, one can increase the pair $(n, m)$ stepwise (according to $\mathfrak{R}$ ) from $(0,0)$, until the condition (18) is satisfied. Then using (19), the coefficients of $\mathbf{p}\left(\lambda_{h}, \lambda_{v}\right)$ can be determined, along with those of $\mathbf{q}\left(\lambda_{h}, \lambda_{v}\right)=\Lambda_{h} \mathbf{Q} \Lambda_{v}^{\top}$, as illustrated with the following toy example. As discussed further in Remark 3.2, the choice of $\mathfrak{R}$ should be made on the basis of the method to be used to construct a latent variable model realization of the resulting rational polynomial model-see Section III-B.

Example 3.1: Let $N=3$ and $M=2$, and consider a Toeplitz kernel $\varphi$ such that

$$
\Phi_{0,0}=\left[\begin{array}{lll}
\varphi_{3,2} & \varphi_{3,1} & \varphi_{3,0} \\
\varphi_{2,2} & \varphi_{2,1} & \varphi_{2,0} \\
\varphi_{1,2} & \varphi_{1,1} & \varphi_{1,0} \\
\varphi_{0,2} & \varphi_{0,1} & \varphi_{0,0}
\end{array}\right]=\left[\begin{array}{ccc}
9.75 & -5.5 & 2 \\
-5.625 & 4 & -2 \\
2.625 & -2.5 & 2 \\
-0.75 & 1 & -2
\end{array}\right] .
$$

Consider also the ordering $\dot{\leq}$ on $[0, N] \times[0, M] \backslash\{(0,0)\}$ defined by

$$
\begin{gathered}
(1,0) \dot{\leq}(0,1) \dot{\leq}(2,0) \dot{\leq}(1,1) \dot{\leq}(0,2) \dot{\leq}(3,0) \dot{\leq} \\
\dot{\leq}(2,1) \dot{\leq}(1,2) \dot{\leq}(3,1) \dot{\leq}(2,2) \dot{\leq}(3,2) .
\end{gathered}
$$

${ }^{2}$ Ordered conformably with $H_{[n, m]}\left(k_{0}, l_{0}\right)$.
Begin by testing the condition (18) of Theorem 3.1 for $(n, m)=$ $(1,0)$ : (14) becomes

$$
\begin{gathered}
{\left[\begin{array}{ccc}
9.75 & -5.5 & 2 \\
-5.625 & 4 & -2 \\
2.625 & -2.5 & 2 \\
-0.75 & 1 & -2
\end{array}\right]+p_{1,0}\left[\begin{array}{ccc}
-5.625 & 4 & -2 \\
2.625 & -2.5 & 2 \\
-0.75 & 1 & -2 \\
0 & 0 & 0
\end{array}\right]} \\
=\left[\begin{array}{ccc}
0 & 0 & 0 \\
0 & 0 & 0 \\
0 & 0 & \omega_{1} \\
0 & 0 & \omega_{2}
\end{array}\right]
\end{gathered}
$$

where $\Omega=\left[\begin{array}{c}\omega_{1} \\ \omega_{2}\end{array}\right]$, in the unknown $p_{1,0}$. In this case $k_{0}=N-$ $n-1=1, l_{0}=M-m-1=1$. It is easily seen that there are no values of the coefficient $p_{1,0}$ such that (20) holds for some $\omega_{1}, \omega_{2}$ and, in fact, the vector

$$
\begin{aligned}
h^{(0,0)} & (1,1) \\
= & {\left[\begin{array}{l}
S^{(0,0)}(1) \\
C^{(0,0)}(1)
\end{array}\right] } \\
= & {\left[\begin{array}{llllllll}
9.75 & -5.5 & 2 & -5.625 & 4 & -2 & 9.75 & \\
& -5.625 & 2.625 & -0.75 & -5.5 & 4 & -2.5 & 1
\end{array}\right]^{\top} }
\end{aligned}
$$

does not lie in the image of

$$
\begin{aligned}
& H_{[1,0]}(1,1) \\
& =\left[\begin{array}{l}
S^{(1,0)}(1) \\
C^{(1,0)}(1)
\end{array}\right] \\
& =\left[\begin{array}{llllll}
-5.625 & 4 & -2 & 2.625 & -2.5 & 2
\end{array}\right. \\
& \left.\begin{array}{llllllll}
-5.625 & 2.625 & -0.75 & 0 & 4 & -2.5 & 1 & 0
\end{array}\right]^{\top} \text {. }
\end{aligned}
$$

Increasing $n$ and $m$ according to $\dot{\leq}$, the first pair $(n, m)$ for which condition (18) holds is $(1,1)$ : now $k_{0}=N-n-1=$ $1, l_{0}=M-m-1=0$ and condition (18) is satisfied because

$$
h^{(0,0)}(1,0)=S^{(0,0)}(0) \in \operatorname{im} H_{[1,1]}(0,-1)
$$

where

$$
H_{[1,1]}(0,-1)=\left[S^{(0,1)}(0) \quad S^{(1,0)}(0) \quad S^{(1,1)}(0)\right] .
$$

From the solution $\pi=\left[\begin{array}{lll}p_{1,0} & p_{0,1} & p_{1,1}\end{array}\right]^{\top}=$ $-H_{[1,1]}(1,0)^{\dagger} h^{(0,0)}(1,0)=\left[\begin{array}{lll}0.75 & 1 & 0\end{array}\right]^{\uparrow}$ it follows

$$
\begin{aligned}
& S^{(i, j)}(k):=\left[\begin{array}{llll}
R_{0}^{(i, j)} & R_{1}^{(i, j)} & \ldots & R_{k}^{(i, j)}
\end{array}\right]^{\top} \\
& C^{(i, j)}(l):=\left[\begin{array}{llll}
\left(C_{0}^{(i, j)}\right)^{\top} & \left(C_{1}^{(i, j)}\right)^{\top} & \ldots & \left(C_{l}^{(i, j)}\right)^{\top}
\end{array}\right]^{\top} \\
& h^{(0,0)}(k, l):=\left\{\begin{array}{lll}
{\left[\begin{array}{l}
S^{(0,0)}(k) \\
C^{(0,0)}(l)
\end{array}\right],} & \text { if } k \geq 0 & \text { and } l \geq 0 \\
S^{(0,0)}(k), & \text { if } k \geq 0 & \text { and } l<0 \\
C^{(0,0)}(l), & \text { if } k<0 & \text { and } l \geq 0
\end{array}\right.
\end{aligned}
$$

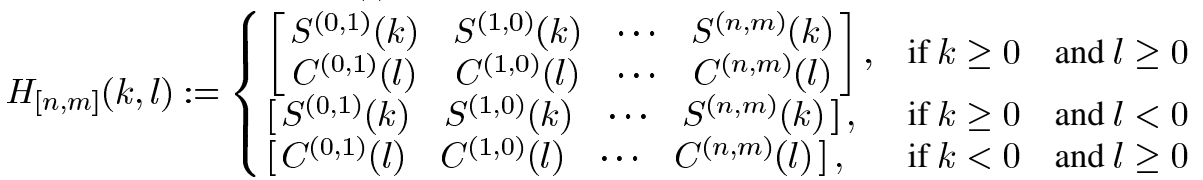


that

$$
\mathbf{Q}=\sum_{i=0}^{2} \sum_{j=0}^{2} p_{i, j} \Phi_{i, j}=\left[\begin{array}{ccc}
0 & 0 & 0 \\
0 & 0 & 0 \\
0 & -0.5 & 0 \\
0 & 0 & -2
\end{array}\right] .
$$

That is, $\mathbf{p}\left(\lambda_{h}, \lambda_{v}\right)=1+0.75 \lambda_{h}+\lambda_{v}$ and $\mathbf{q}\left(\lambda_{h}, \lambda_{v}\right)=-2-$ $0.5 \lambda_{h} \lambda_{v}$ solve the corresponding Problem 3.1.

Remark 3.1: In the algorithm described above, when a pair $(n, m)$ is found such that condition (18) is satisfied with $k_{0}=N-n-1$ and $l_{0}=M-m-1$, the degrees of the resulting polynomials $\mathbf{p}\left(\lambda_{h}, \lambda_{v}\right)$ and $\mathbf{q}\left(\lambda_{h}, \lambda_{v}\right)$ are both $(n, m)$. There are cases when it is possible to find a polynomial $\mathbf{q}\left(\lambda_{h}, \lambda_{v}\right)$ whose degree $\left(n_{q}, m_{q}\right)$ is lower than $(n, m)$. In more precise terms, given $n_{q} \leq n$ and $m_{q} \leq m$, a necessary and sufficient condition for the existence of a polynomial $\mathbf{p}\left(\lambda_{h}, \lambda_{v}\right)=\sum_{i=0}^{n} \sum_{j=0}^{m} p_{i, j} \lambda_{h}^{i} \lambda_{v}^{j}$, with $p_{0,0}=1$, and a polynomial $\mathbf{q}\left(\lambda_{h}, \lambda_{v}\right)=\sum_{i=0}^{n_{q}} \sum_{j=0}^{m_{q}} q_{i, j} \lambda_{h}^{i} \lambda_{v}^{j}$, which together partially realize the kernel $\varphi$, is that condition (18) hold with $k_{0}=N-n_{q}-1$ and $l_{0}=M-m_{q}-1$.

\section{B. Latent Variable Model Realization of Rational Polynomial Models}

In the preceding section an algorithm is proposed for constructing a rational polynomial model to partially realize a given sw-causal Toeplitz kernel. Here it is shown how to construct a (recursive) NNM given such a realization. The NNM [16] is chosen in view of the fact that the NNMs obtained by realising the quarter-plane causal components of an originally noncausal Toeplitz kernel can be combined, as described in Section IV, into a single NNM in the form

$x_{i, j}=A_{1} x_{i-1, j}+A_{2} x_{i+1, j}+A_{3} x_{i, j-1}+A_{4} x_{i, j+1}+B u_{i, j}$ $y_{i, j}=C x_{i, j}$

with appropriately assigned boundary conditions. That is, the class of NNMs is closed under the manipulations employed in this paper. The same is true [21] for the class of generalized FM models in implicit form, proposed by Kaczorek [12].

Consider the simplified NNM

$$
\begin{aligned}
x_{i, j} & =A_{1} x_{i-1, j}+A_{2} x_{i, j-1}+B u_{i, j}, \quad i, j \in \mathbb{N} \times \mathbb{N} \\
y_{i, j} & =C x_{i, j}
\end{aligned}
$$

with boundary conditions $x_{i,-1}=x_{-1, j}=0$ for all $i, j \geq 0$, where $A_{1}, A_{2} \in \mathbb{R}^{n \times n}, B \in \mathbb{R}^{n \times 1}$ and $C \in \mathbb{R}^{1 \times n}$. Observe that this recursive model is capable of producing sw-causal input-output behavior. Moreover, with the boundary conditions set as shown, it follows that the formal power series representations of the signals $u: \mathbb{N} \times \mathbb{N} \rightarrow \mathbb{R}$ and $y: \mathbb{N} \times \mathbb{N} \rightarrow \mathbb{R}$ are related by

where

$$
\mathbf{y}\left(\lambda_{h}, \lambda_{v}\right)=C\left(I-A_{1} \lambda_{h}-A_{2} \lambda_{v}\right)^{-1} B \mathbf{u}\left(\lambda_{h}, \lambda_{v}\right)
$$

$$
\left(I-A_{1} \lambda_{h}-A_{2} \lambda_{v}\right)^{-1}=\sum_{i=0}^{\infty}\left(A_{1} \lambda_{h}+A_{2} \lambda_{v}\right)^{i}
$$

denotes the inverse of $\left(I-A_{1} \lambda_{h}-A_{2} \lambda_{v}\right)$ in the ring of formal power series in the indeterminates $\lambda_{h}$ and $\lambda_{v}$.

Definition 3.1: The quadruple $\left(A_{1}, A_{2}, B, C\right)$, and hence the corresponding NNM (22), is said to be a realization of a rational polynomial model $\mathbf{p}\left(\lambda_{h}, \lambda_{v}\right)$ and $\mathbf{q}\left(\lambda_{h}, \lambda_{v}\right)$ in the form (7)-(8), if $\mathbf{p}\left(\lambda_{h}, \lambda_{v}\right) G\left(\lambda_{h}, \lambda_{v}\right)=\mathbf{q}\left(\lambda_{h}, \lambda_{v}\right)$, where $G\left(\lambda_{h}, \lambda_{v}\right)=C\left(I-A_{1} \lambda_{h}-A_{2} \lambda_{v}\right)^{-1} B$.
Theorem 3.2: A rational polynomial model $\mathbf{p}\left(\lambda_{h}, \lambda_{v}\right)$ and $\mathbf{q}\left(\lambda_{h}, \lambda_{v}\right)$ can be realized by a suitably defined quadruple $\left(A_{1}, A_{2}, B, C\right)$-see (24) below.

Proof: As shown in [7], [1], a rational polynomial model $\mathbf{p}\left(\lambda_{h}, \lambda_{v}\right)$ and $\mathbf{q}\left(\lambda_{h}, \lambda_{v}\right)$ of the form (7)-(8) admits a realization in the form of a first-order FM model

$$
\begin{aligned}
\xi_{i+1, j+1} & =F_{1} \xi_{i+1, j}+F_{2} \xi_{i, j+1}+G_{1} u_{i+1, j}+G_{2} u_{i, j+1} \\
y_{i, j} & =H \xi_{i, j}+J u_{i, j}, \quad i, j \in \mathbb{N} \times \mathbb{N}
\end{aligned}
$$

with boundary conditions $\xi_{i, 0}=\xi_{0, j}=0$ for $i, j \in \mathbb{N}$, where

$$
\begin{aligned}
& F_{1}=\left[\begin{array}{lllll}
\ddots & & & & \\
& & & & \\
& 0 & 0 & 0 & 0 \\
0 & 0 & 0 & 0 \\
& 0 & 0 & 0 & 1
\end{array}\right. \\
& \begin{array}{lll}
0 & 0 & 0 \\
0 & 0 & 1
\end{array} \\
& \begin{array}{lll}
0 & 0 & 1
\end{array} \\
& \left.\begin{array}{lll}
0 & 1 & -p_{1,0}
\end{array}\right] \\
& {\left[\begin{array}{l} 
\\
\ddots
\end{array}\right.} \\
& \begin{array}{c}
\vdots \\
-p_{0,4}
\end{array} \\
& -p_{1,3} \\
& -p_{2,2} \\
& -p_{3,1} \\
& -p_{0,3} \\
& \begin{array}{llll}
1 & 0 & 0 & 0 \\
0 & 1 & 0 & 0
\end{array} \\
& \begin{array}{llll}
0 & 1 & 0 & 0
\end{array} \\
& \begin{array}{llll}
0 & 0 & 1 & 0
\end{array} \\
& -p_{1,2} \\
& -p_{2,1} \\
& \begin{array}{lll}
1 & 0 & 0
\end{array} \\
& -p_{0,2} \\
& \begin{array}{lll}
0 & 1 & 0
\end{array} \\
& -p_{1,1} \\
& \begin{array}{lll}
1 & 0 & -p_{0,1}
\end{array}
\end{aligned}
$$


That is, $F_{1}, F_{2}, G_{1}, G_{2}, H, J$ are known to exist such that

$$
\begin{aligned}
\mathbf{p}\left(\lambda_{h}, \lambda_{v}\right)\left(H\left(I-F_{1} \lambda_{h}-F_{2} \lambda_{v}\right)^{-1}\left(G_{1} \lambda_{h}+G_{2} \lambda_{v}\right)\right. & +J) \\
& =\mathbf{q}\left(\lambda_{h}, \lambda_{v}\right) .
\end{aligned}
$$

Now, with

$$
\begin{aligned}
A_{1} & :=\left[\begin{array}{cc}
0 & 0 \\
G_{2} & F_{2}
\end{array}\right] \\
A_{2} & :=\left[\begin{array}{cc}
0 & 0 \\
G_{1} & F_{1}
\end{array}\right] \\
B & :=\left[\begin{array}{l}
1 \\
0
\end{array}\right] \\
C & :=\left[\begin{array}{ll}
J & H
\end{array}\right]
\end{aligned}
$$

it follows that

$$
\begin{aligned}
\left(H ( I - F _ { 1 } \lambda _ { h } - F _ { 2 } \lambda _ { v } ) ^ { - 1 } \left(G_{1} \lambda_{h}\right.\right. & \left.\left.+G_{2} \lambda_{v}\right)+J\right) \\
& =C\left(I-A_{1} \lambda_{h}-A_{2} \lambda_{v}\right)^{-1} B
\end{aligned}
$$

and hence, that the quadruple $\left(A_{1}, A_{2}, B, C\right)$ realizes $\mathbf{p}\left(\lambda_{h}, \lambda_{v}\right)$ and $\mathbf{q}\left(\lambda_{h}, \lambda_{v}\right)$, as required.

Example 3.2: The polynomials $\mathbf{p}\left(\lambda_{h}, \lambda_{v}\right)=1+2 \lambda_{h}+\lambda_{v}+$ $3 \lambda_{h} \lambda_{v}$ and $\mathbf{q}\left(\lambda_{h}, \lambda_{v}\right)=2+5 \lambda_{h}+\lambda_{v}+2 \lambda_{h} \lambda_{v}$ can be realized in terms of a FM model (23) with (see, e.g., [1])

$$
\begin{aligned}
F_{1} & =\left[\begin{array}{lll}
0 & 0 & 0 \\
0 & 0 & -3 \\
1 & 0 & -1
\end{array}\right] \\
F_{2} & =\left[\begin{array}{lll}
0 & 0 & 0 \\
0 & 0 & 0 \\
0 & 1 & -2
\end{array}\right] \\
G_{1} & =\left[\begin{array}{c}
0 \\
-4 \\
-1
\end{array}\right] \\
G_{2} & =\left[\begin{array}{l}
0 \\
0 \\
1
\end{array}\right] \\
H & =\left[\begin{array}{lll}
0 & 0 & 1
\end{array}\right] \\
J & =2 .
\end{aligned}
$$

The corresponding recursive NNM realization is characterized by the matrices

$$
\begin{aligned}
& A_{1}=\left[\begin{array}{cccc}
0 & 0 & 0 & 0 \\
0 & 0 & 0 & 0 \\
0 & 0 & 0 & 0 \\
1 & 0 & 1 & -2
\end{array}\right] \\
& A_{2}=\left[\begin{array}{cccc}
0 & 0 & 0 & 0 \\
0 & 0 & 0 & 0 \\
-4 & 0 & 0 & -3 \\
-1 & 1 & 0 & -1
\end{array}\right] \\
& B=\left[\begin{array}{l}
1 \\
0 \\
0 \\
0
\end{array}\right] \\
& C=\left[\begin{array}{llll}
2 & 0 & 0 & 1
\end{array}\right] \text {. }
\end{aligned}
$$

Remark 3.2: Given a rational polynomial model of the form (7)-(8) with degree $(n, m)$, the square matrices $F_{1}$ and $F_{2}$ in the particular latent variable model realization used to prove Theorem 3.2, have column/row dimension $s(s+1) / 2$, where $s=n+m$. As such, it would seem appropriate to choose the ordering $\mathfrak{R}$ in the algorithm proposed for constructing a rational polynomial model for a sw-causal Toeplitz kernel based on Theorem 3.1 , such that $(i, j) \dot{\leq}(k, l)$ for $i+j \leq k+l .{ }^{3}$ Indeed, with such an ordering, the algorithm yields a realization that is minimal in the (weak) sense that it produces a rational polynomial model for which the "dimension" of the particular latent variable model realization used in the proof of Theorem 3.2, is smallest.

\section{LATENT VARIABLE MODEL REALIZATION OF NONCAUSAL TOEPLITZ KERNELS}

In Section II, it is shown how to linearly decompose the input-output model (1), with noncausal Toeplitz kernel, into four quarter-plane causal components. By transformation of the spatial indexes, input, output and kernel, each component is then shown to be equivalent to a suitably defined sw-causal system $\hat{\mathcal{F}}^{\Delta}: \hat{u}^{\Delta} \mapsto \hat{y}^{\Delta}$ governed by (5). Applying the results of Section III to each sw-causal system $\hat{\mathcal{F}}^{\Delta}$, yields a rational polynomial realization $\hat{\mathbf{p}}^{\Delta}\left(\lambda_{h}, \lambda_{v}\right)$ and $\hat{\mathbf{q}}^{\Delta}\left(\lambda_{h}, \lambda_{v}\right)$, and a corresponding (recursive) NMM realization $\left(A_{1}^{\Delta}, A_{2}^{\Delta}, B^{\Delta}, C^{\Delta}\right)$, for the transformed kernel $\hat{\varphi}^{\Delta}$. The aim now is to combine these models to obtain a latent variable model to realize the 2 -D process (1), over the bounded frame $[0, N] \times[0, M]$. This involves inversion of the spatial index transformations used to obtain each $\hat{\varphi}^{\Delta}$, and yields a NNM in the general form (21), [16], with the appropriately defined latent variable boundary values over the bounded frame of interest.

More explicitly, by exploiting for example the realization presented in Subsection III.B, for each pair of polynomials $\hat{\mathbf{p}}^{\Delta}\left(\lambda_{h}, \lambda_{v}\right)$ and $\hat{\mathbf{q}}^{\Delta}\left(\lambda_{h}, \lambda_{v}\right)$, matrices $A_{1}^{\Delta}, A_{2}^{\Delta} B^{\Delta}$ and $C^{\Delta}$ can be determined so that the sw-causal (and hence, recursive) NNM

$$
\begin{aligned}
\hat{x}_{i_{\Delta}, j_{\Delta}}^{\Delta} & =A_{1}^{\Delta} \hat{x}_{i_{\Delta}-1, j_{\Delta}}^{\Delta}+A_{2}^{\Delta} \hat{x}_{i_{\Delta}, j_{\Delta}-1}^{\Delta}+B^{\Delta} \hat{u}_{i_{\Delta}, j_{\Delta}}^{\Delta} \\
\hat{z}_{i_{\Delta}, j_{\Delta}}^{\Delta} & =C^{\Delta} \hat{x}_{i_{\Delta}, j_{\Delta}}^{\Delta}
\end{aligned}
$$

with boundary conditions $\hat{x}_{i_{\Delta},-1}^{\Delta}=0$ and $\hat{x}_{-1, j_{\Delta}}^{\Delta}=0$ for $i_{\Delta}, j_{\Delta} \in \mathbb{N}$, satisfies

$$
\hat{\mathbf{p}}^{\Delta}\left(\lambda_{h}, \lambda_{v}\right) \hat{\mathbf{z}}^{\Delta}\left(\lambda_{h}, \lambda_{v}\right)=\hat{\mathbf{q}}^{\Delta}\left(\lambda_{h}, \lambda_{v}\right) \hat{\mathbf{u}}^{\Delta}\left(\lambda_{h}, \lambda_{v}\right)
$$

and hence, $\Pi_{[0, N] \times[0, M]}\left(\hat{\mathbf{z}}^{\Delta}\left(\lambda_{h}, \lambda_{v}\right)\right)=\hat{\mathbf{y}}^{\Delta}\left(\lambda_{h}, \lambda_{v}\right)$-i.e., the NNM (25) partially realizes $\hat{\mathcal{F}}^{\Delta}$. Now inverting the spatial index transformations described at the end of Section 2 (see Tables I and II), gives the following NNM model for a partial realization of each $\Delta$-causal $\mathcal{F}^{\Delta}$ component:

$$
\begin{aligned}
x_{i, j}^{\Delta} & =A_{1}^{\Delta} x_{\Phi^{\Delta}}^{\Delta}+A_{2}^{\Delta} x_{\Psi^{\Delta}}^{\Delta}+B^{\Delta} u_{i, j} \\
z_{i, j}^{\Delta} & =C^{\Delta} x_{i, j}^{\Delta}
\end{aligned}
$$

with boundary conditions $x_{h^{\Delta}, j}^{\Delta}=0$ for $j \in I_{v}^{\Delta}$ and $x_{i, v^{\Delta}}^{\Delta}=0$ for $i \in I_{h}^{\Delta}$, where $I_{h}^{\Delta}, I_{v}^{\Delta}, h^{\Delta}, v^{\Delta}$ and the double index symbols $\Phi^{\Delta}$ and $\Psi^{\Delta}$ are defined in Table III. For example, given a NNM realization $\left(A_{1}^{\mathrm{nw}}, A_{2}^{\mathrm{nw}}, B^{\mathrm{nw}}, C^{\mathrm{nw}}\right)$ for the sw-causal $\hat{\mathcal{F}}^{\text {nw }}$, the NNM

$$
\begin{aligned}
x_{i, j}^{\mathrm{nw}} & =A_{1}^{\mathrm{nw}} x_{i-1, j}^{\mathrm{nw}}+A_{2}^{\mathrm{nw}} x_{i, j+1}^{\mathrm{nw}}+B^{\mathrm{nw}} u_{i, j} \\
z_{i, j}^{\mathrm{nw}} & =C^{\mathrm{nw}} x_{i, j}^{\mathrm{nw}}
\end{aligned}
$$

${ }^{3}$ This is in fact the ordering used (without justification) in the related work [24] on 2-D realization. 
TABLE III

INTERVALS AND SIGN CONSTANTS

\begin{tabular}{|c||c||c|c||c|c|}
\hline$\Delta$ & $I_{h}^{\Delta} \times I_{v}^{\Delta}$ & $h^{\Delta}$ & $v^{\Delta}$ & $\Phi^{\Delta}$ & $\Psi^{\Delta}$ \\
\hline \hline sw & {$[0, \infty) \times[0, \infty)$} & -1 & -1 & $i-1, j$ & $i, j-1$ \\
\hline nw & {$[0, \infty) \times(-\infty, M]$} & -1 & $M+1$ & $i-1, j$ & $i, j+1$ \\
\hline ne & $(-\infty, N] \times(-\infty, M]$ & $N+1$ & $M+1$ & $i+1, j$ & $i, j+1$ \\
\hline se & $(-\infty, N] \times[0, \infty)$ & $N+1$ & -1 & $i+1, j$ & $i, j-1$ \\
\hline
\end{tabular}

with boundary conditions $x_{-1, j}^{\mathrm{nw}}=0$ for all $j \geq 0$ and $x_{i, M+1}^{\mathrm{nw}}=0$ for all $i \geq 0$, which clearly exhibits nw causal input-output behavior, partially realizes the nw-causal component of the Toeplitz kernel.

Now, defining the latent variable $x_{i, j}:=$ $\left[\begin{array}{llll}x_{i, j}^{\mathrm{sw}}{ }^{\mathrm{s}} & x_{i, j}^{\mathrm{nw} \top} & x_{i, j}^{\mathrm{ne} \top} & x_{i, j}^{\mathrm{se} \top}\end{array}\right]^{\top}$ and the output $z_{i, j}:=$ $z_{i, j}^{\mathrm{sw}}+z_{i, j}^{\mathrm{nw}}+z_{i, j}^{\mathrm{ne}}+z_{i, j}^{\mathrm{se}}$, it is straightforward to verify that, over the bounded frame $[0, N] \times[0, M]$, equations (26)-(27) are completely characterized by the following NNM:

$$
\begin{aligned}
x_{i, j}= & A_{1} x_{i-1, j}+A_{2} x_{i+1, j}+A_{3} x_{i, j-1} \\
& +A_{4} x_{i, j+1}+B u_{i, j} \\
z_{i, j}= & C x_{i, j}
\end{aligned}
$$

with boundary conditions

$$
\begin{aligned}
{\left[\begin{array}{llll}
I & 0 & 0 & 0
\end{array}\right] x_{i,-1} } & =\left[\begin{array}{llll}
I & 0 & 0 & 0
\end{array}\right] x_{-1, j}=0 \\
{\left[\begin{array}{llll}
0 & I & 0 & 0
\end{array}\right] x_{i, M+1} } & =\left[\begin{array}{llll}
0 & I & 0 & 0
\end{array}\right] x_{-1, j}=0 \\
{\left[\begin{array}{llll}
0 & 0 & I & 0
\end{array}\right] x_{i, M+1} } & =\left[\begin{array}{llll}
0 & 0 & I & 0
\end{array}\right] x_{N+1, j}=0 \\
{\left[\begin{array}{llll}
0 & 0 & 0 & I
\end{array}\right] x_{i,-1} } & =\left[\begin{array}{llll}
0 & 0 & 0 & I
\end{array}\right] x_{N+1, j}=0
\end{aligned}
$$

for $(i, j) \in[0, N] \times[0, M]$ on the latent variable, where

$$
\begin{aligned}
& A_{1}:=\left[\begin{array}{cccc}
A_{1}^{\mathrm{sw}} & 0 & 0 & 0 \\
0 & A_{1}^{\mathrm{nw}} & 0 & 0 \\
0 & 0 & 0 & 0 \\
0 & 0 & 0 & 0
\end{array}\right] \\
& A_{2}:=\left[\begin{array}{cccc}
0 & 0 & 0 & 0 \\
0 & 0 & 0 & 0 \\
0 & 0 & A_{1}^{\text {ne }} & 0 \\
0 & 0 & 0 & A_{1}^{\text {se }}
\end{array}\right] \\
& A_{3}:=\left[\begin{array}{cccc}
A_{2}^{\mathrm{sw}} & 0 & 0 & 0 \\
0 & 0 & 0 & 0 \\
0 & 0 & 0 & 0 \\
0 & 0 & 0 & A_{2}^{\mathrm{se}}
\end{array}\right] \\
& A_{4}:=\left[\begin{array}{cccc}
0 & 0 & 0 & 0 \\
0 & A_{2}^{\text {nw }} & 0 & 0 \\
0 & 0 & A_{2}^{\text {ne }} & 0 \\
0 & 0 & 0 & 0
\end{array}\right] \\
& B:=\left[\begin{array}{l}
B^{\mathrm{sw}} \\
B^{\mathrm{nw}} \\
B^{\mathrm{ne}} \\
B^{\mathrm{se}}
\end{array}\right] \\
& C:=\left[\begin{array}{llll}
C^{\mathrm{sw}} & C^{\mathrm{nw}} & C^{\mathrm{ne}} & C^{\mathrm{se}}
\end{array}\right] .
\end{aligned}
$$

Note that in this latent variable model the boundary conditions on the local state $x$ are given on each side of the bounded frame $[0, N] \times[0, M]$.

\section{CONCLUSION}

The problem of partial realization is considered for bounded frame noncausal 2-D processes, in terms of a latent variable model in form of the so-called NNM. The key idea is to decom- pose the Toeplitz kernel of the given noncausal process into four components, each displaying a particular quarter-plane causal structure. These components are then partially realized separately in terms of recursive forms of the NNM. It is then shown how to combine these realizations into a single NNM in its general form, with latent variable boundary values assigned around the boundary of the frame of interest. The final model essentially comprises four components, each with recursively computable structure. An interesting related open problem is as follows: Does there exist a transformation of an arbitrary NNM model $\left(A_{1}, A_{2}, A_{3}, A_{4}, B, C\right)$, which yields four recursively computable parts, in a manner similar to the case of implicit 1-D models, as demonstrated in [18]?

\section{REFERENCES}

[1] M. Bisiacco, "Stabilization theory for single-input/single-output twodimensional systems," Circuits Syst. Signal Process., vol. 6, no. 1, pp. 77-93, 1987.

[2] M. Bisiacco, E. Fornasini, and G. Marchesini, "Dynamic regulation of 2-D Systems: A state-space approach," Linear Algebra Appl., vol. 122-124, pp. 195-218, 1989.

[3] N. K. Bose, Multidimensional Systems Theory and Applications. Dordrecht, The Netherlands: Kluwer, 2003.

[4] R. Eising, "Realization and stabilization of 2-D systems," IEEE Trans. Autom. Contr., vol. AC-23, no. 5, pp. 793-799, Oct. 1978

[5] R. Eising, "State-space realization and inversion of 2-D systems," IEEE Trans. Circuits Syst., vol. CAS-27, no. 7, pp. 612-619, Jul. 1980.

[6] E. Fornasini and G. Marchesini, "State-space realization theory of twodimensional filters," IEEE Trans. Autom. Contr., vol. AC-21, no. 4, pp. 484-492, Aug. 1976.

[7] E. Fornasini and G. Marchesini, "Doubly-indexed dynamical systems: State-space models and structural properties," Math. Syst. Theory, vol 12, pp. 59-72, 1978.

[8] E. Fornasini, P. Rocha, and S. Zampieri, "State space realization of 2-D finite-dimensional behaviors," SIAM J. Contr. Optim., vol. 31, no. 6, pp. 1502-1517, Nov. 1993.

[9] W. B. Gragg and A. Lindquist, "On the partial realization problem," Linear Algebra Appl., vol. 50, pp. 277-319, 1983.

[10] T. Hinamoto, "Realizations of a state-space model from two-dimensional input-output map," IEEE Trans. Circuits Syst., vol. CAS-27, no. 1, pp. 36-44, Jan. 1980.

[11] A. K. Jain, "Advances in mathematical models for image processing," Proc. IEEE, vol. 69, no. 5, pp. 502-528, May 1981.

[12] T. Kaczorek, "Singular general model of 2-D systems and its solution," IEEE Trans. Autom. Contr., vol. 33, no. 11, pp. 1060-1061, Nov. 1988.

[13] Y. Kao and C. Chen, "Two-dimensional Hankel theory," Int. J. Contr., vol. 31, no. 2, pp. 271-284, 1980.

[14] S.-Y. Kung, B. C. Levy, M. Morf, and T. Kailath, "New results in 2-D systems theory, Part I: 2-D state-space models-Realization and the notions of controllability, observability and minimality," Proc. IEEE, vol. 65 , no. 6, pp. 945-961, Jun. 1977.

[15] J. Kurek, "The general state-space model for a two-dimensional linear digital system," IEEE Trans. Autom. Contr., vol. AC-30, no. 6, pp. 600-602, Jun. 1985.

[16] B. C. Levy, M. B. Adams, and A. S. Willsky, "Solution and linear estimation of 2-D nearest-neighbor models," Proc. IEEE, vol. 78, no. 4, pp. 627-641, Apr. 1990.

[17] F. Lewis, "A review of 2-D implicit systems," Automatica, vol. 28, no. 2, pp. 345-354, 1992.

[18] F. Lewis, "Descriptor systems: Decomposition into forward and backward subsystems," IEEE Trans. Autom. Contr., vol. AC-29, no. 2, pp. 167-170, Feb. 1984 
[19] F. Lewis and B. G. Mertzios, "On the analysis of two-dimensional discrete singular systems," Circuits Syst. Signal Process., vol. 11, no. 3, pp. 399-419, 1992.

[20] S. Mac Lane and G. Birkhoff, Algebra. Providence, R.I.: AMS, 1999.

[21] L. Ntogramatzidis, M. Cantoni, and R. Yang, "On the partial realization of noncausal 2-D linear systems," in Proc. 17th Int. Symp. Math. Theory Networks Syst. (MTNS 06), Kyoto, Japan, Jul. 24-28, 2006.

[22] J. W. Polderman and J. C. Willems, Introduction to Mathematical Systems Theory: A Behavioral Approach. New York: Springer-Verlag, 1997.

[23] R. Roesser, "A discrete state-space model for linear image processing," IEEE Trans. Autom. Contr., vol. AC-20, no. 1, pp. 1-10, Feb. 1975.

[24] S. Sakata, "On the minimal partial realization of 2-D discrete linear shift-invariant systems," IEEE Trans. Autom. Contr., vol. 36, no. 8, pp. 984-988, Aug. 1991.

[25] Y. Uetake, "Realization of noncausal 2-D systems based on a descriptor model," IEEE Trans. Autom. Contr., vol. 37, no. 11, pp. 1837-1840, Nov. 1992.

[26] E. Zerz, "First-order representations of discrete linear multidimensional systems," Multidim. Syst, Signal Process., vol. 11, pp. 359-380, 2000.

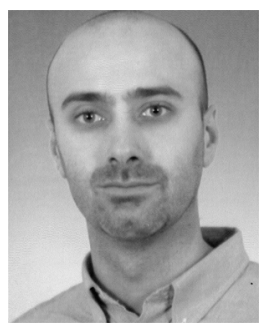

Lorenzo Ntogramatzidis received the "laurea" degree (cum laude), in computer engineering and the Ph.D. degree in automatic control and operations research from the University of Bologna, Bologna, Italy, in 2001 and 2005, respectively.

Since 2005, he has been with the Department of Electrical and Electronic Engineering, The University of Melbourne, Melbourne, Australia, where is currently a Post-Doctoral Research Fellow. His research interests are in the area of systems and control theory.

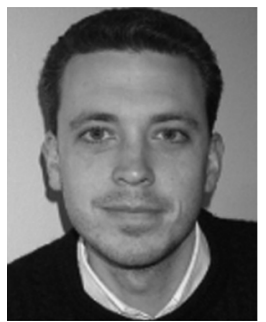

Michael Cantoni (M'00) received the B.E. (Hons. I) degree in electrical engineering and the B.Sc. degree in applied mathematics from the University of Western Australia, Crawley, Australia, in 1995, and the Ph.D. degree in engineering from the University of Cambridge, Cambridge, U.K., in 1998.

From 1998 to 2000, he was a Research Associate with the Department of Engineering at The University of Cambridge and a Junior Research Fellow with St. John's College, Cambridge, U.K. Since 2000, he has been with the Department of Electrical and Electronic Engineering, The University of Melbourne, Australia, where is currently an Associate Professor. His research interests lie in the broad area of systems and control theory.

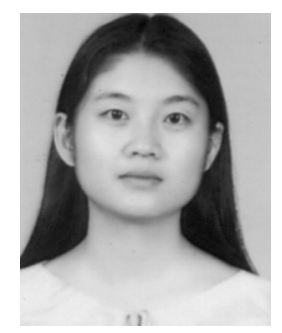

Ran Yang received B.E. degree in electrical engineering from Northeastern University, Shenyang, China, in 1996, and M.Sc. and Ph.D. degrees in applied mathematics and electrical engineering from Shanghai Jiaotong University, Shanghai, China, in 1999 and 2001, respectively.

She worked as a Research Fellow at the University of Melbourne, Melbourne, Australia, from 2001 to 2004. Since 2005, she has been with the School of Information Science and Technology, Sun Yat-Sen University, Guangzhou, China, where she is currently an Associate Professor. Her research interests include multi-dimensional systems, descriptor systems, optimal estimation, robust filtering, and control. 